\title{
Vitamin D bioavailability from different lipid delivery systems
}

\section{Abstract}

Introduction: Data modelling studies confirm a role for food fortification to improve vitamin D intakes and status. However, there is very little research reporting the bioavailability of vitamin $\mathrm{D}$ from foods to inform food fortification strategies. There is some evidence to suggest that the lipid component of foods impacts vitamin D uptake in cells; however, no studies have examined the absorption kinetics of vitamin D from different lipid delivery systems in humans. The aim of this research is to compare changes in serum vitamin $\mathrm{D}$ concentrations using different vitamin $\mathrm{D}$ emulsions as delivery systems.

Methods: A single blind, controlled, 4-way crossover postprandial study was conducted. Participants consumed 4 different $20 \mu \mathrm{g}$ vitamin D enriched emulsion drinks (olive oil emulsion, fish oil emulsion, mixed micelle emulsion and a non-fat control drink) with a low-fat breakfast on 4 occasions separated by a 1-week washout. Serum 25-hydroxyvitamin D $\left(25(\mathrm{OH}) \mathrm{D}_{3}\right)$ concentrations and cardiometabolic risk markers were measured at baseline and 2, 4, 6 and 8 hours after consuming the drink. Statistical analysis was performed in IBM SPSS Statistics, Version 24 (SPSS Inc. Chicago, IL, USA). Repeated measures ANOVA was performed to assess the effects of the 4 treatments.

Results: Six males and six females aged between 22 and 65 years completed the study. All participants were healthy with a mean BMI of $24.7 \pm 4.1 \mathrm{~kg} / \mathrm{m}^{2}$ for males and $25.1 \pm 4.5 \mathrm{~kg} / \mathrm{m}^{2}$ for females. Each $100 \mathrm{ml}$ drink contained $106 \mathrm{kcal}, 4 \mathrm{~g}$ fat, $11.9 \mathrm{~g}$ carbohydrate and $5.5 \mathrm{~g}$ protein, apart from the control which contained $70 \mathrm{kcal}$ and $0 \mathrm{~g}$ fat. The breakfast contained $321 \mathrm{kcal}, 0.7 \mathrm{~g}$ fat, $66 \mathrm{~g}$ carbohydrate and $10.3 \mathrm{~g}$ protein. There was an increase in $25(\mathrm{OH}) \mathrm{D}_{3}$ concentrations over the 8-hour assessment period following consumption of all 4 drinks. Two-way repeated measures ANOVA showed a significant drink*time interaction. No participants experienced any negative side effects after consuming any of the drinks.

Discussion: The absorption kinetics of vitamin $\mathrm{D}$ is different depending on the lipid delivery system. This is the first human study demonstrating the bioavailability of vitamin $\mathrm{D}_{3}$ from various emulsions including a novel mixed micelle emulsion. All emulsions were palatable, effective and safe to consume.

\section{Conflict of Interest}

There is no conflict of interest 